\title{
Behavior of triple Langmuir probes in non-equilibrium plasmas
}

\author{
Kurt A. Polzin* and Alicia C. Ratcliffe ${ }^{\dagger}$ \\ NASA-Marshall Space Flight Center, Huntsville, AL 35812
}

\section{Abstract}

$\mathrm{T}$

HE triple Langmuir probe ${ }^{1}$ is an electrostatic probe in which three probe tips collect current when inserted into a plasma. The triple probe differs from a simple single Langmuir probe in the nature of the voltage applied to the probe tips. In the single probe, a swept voltage is applied to the probe tip to acquire a waveform showing the collected current as a function of applied voltage (I-V curve). In a triple probe three probe tips are electrically coupled to each other with constant voltages applied between each of the tips. The voltages are selected such that they would represent three points on the single Langmuir probe I-V curve. Elimination of the voltage sweep makes it possible to measure time-varying plasma properties in transient plasmas. Under the assumption of a Maxwellian plasma, one can determine the time-varying plasma temperature $T_{e}(t)$ and number density $n_{e}(t)$ from the applied voltage levels and the time-histories of the collected currents.

In the present paper we examine the theory of triple probe operation, specifically focusing on the assumption of a Maxwellian plasma. Triple probe measurements have been widely employed for a number of pulsed and timevarying plasmas, including pulsed plasma thrusters (PPTs), ${ }^{2-4}$ dense plasma focus devices, ${ }^{5}$ plasma flows, ${ }^{6}$ and fusion experiments. ${ }^{7}$ While the equilibrium assumption may be justified for some applications, it is unlikely that it is fully justifiable for all pulsed and time-varying plasmas or for all times during the pulse of a plasma device.

To examine a simple non-equilibrium plasma case, we return to basic governing equations of probe current collection and compute the current to the probes for a distribution function consisting of two Maxwellian distributions with different temperatures (the two-temperature Maxwellian). A variation of this method is also employed, where one of the Maxwellians is offset from zero (in velocity space) to add a suprathermal beam of electrons to the tail of the main Maxwellian distribution (the bump-on-the-tail distribution function). For a range of parameters in these non-Maxwellian distributions, we compute the current collection to the probes. We compare the distribution function that was assumed a priori with the distribution function one would infer when applying standard triple probe theory to analyze the collected currents. For the assumed class of non-Maxwellian distribution functions this serves to illustrate the effect a non-Maxwellian plasma would have on results interpreted using the equilibrium triple probe current collection theory, allowing us to state the magnitudes of these deviations as a function of the assumed distribution function properties.

\section{References}

${ }^{1}$ S.-L. Chen and T. Sekiguchi, "Instantaneous direct-display system of plasma parameters by means of triple probe," J. Appl. Phys., Vol. 36, No. 8, 2363-2375 (1965).

${ }^{2}$ R. Eckman, L. Byrne, N.A. Gatsonis, and E. Pencil, "Triple Langmuir probe measurements in the plume of a pulsed plasma thruster," $J$. Propuls. Power, Vol. 17, No. 4, 762-771 (2001).

${ }^{3}$ N.A. Gatsonis, L.T. Byrne, J. Zwahlen, E.J. Pencil, and H. Kamhawi, "Current-mode triple and quadruple Langmuir probe methods with applications to flowing pulsed plasmas," IEEE Trans. Plasma Sci., Vol. 32, No. 5, 2118-2129 (2004).

${ }^{4}$ M.S. Glascock, J.L. Rovey, S. Williams, and J. Thrasher, "Plume characterization of electric solid propellant pulsed microthrusters," $J$. Propuls. Power, In Press (2017).

${ }^{5}$ M. Martinez-Fuentes, H. Ortiz-Uribe, F. Castillo-Mejía, M. Nieto-Pérez, J.J.E. Herrera-Velázquez, and J. Rangel-Gutiérrez, "Dynamics of the expansion discharge originated by a dense plasma focus," J. Phys.: Conf. Series, Vol. 370, No. 1, 012059 (2012).

${ }^{6}$ T.K. Gray, M.J. Williams, and D.N. Ruzic, "Overview and status of the Divertor Erosion and Vapor shielding eXperiment (DEVeX)," 2007 IEEE 22nd Symposium on Fusion Engineering, Albuquerque, NM, 17-21 June 2007.

\footnotetext{
*Associate Fellow AIAA.

${ }^{\dagger}$ Student Member AIAA.
} 
${ }^{7}$ A. Qayyum, S. Ahmad, R. Khan, S. Hussain, F. Deeba, M.A. Javed, N. Ahmed, R. Ali, S. Mehmood, "Triple-probe Diagnostic Measurements in Plasma of GLAST Spherical Tokamak," J. Fusion Energy, Vol. 35, No. 2, 205-213 (2016). 